\title{
Incidence and predictors of asthma exacerbations in middle- aged and older adults: the Rotterdam Study
}

\author{
Emmely W. de Roos $\mathbb{1}^{1,2}$, Lies Lahousse $\mathbb{B}^{2,3}$, Katia M.C. Verhamme $\mathbb{1}^{3,4}$, Gert-Jan Braunstahl $\mathbb{B}^{5,6}$, \\ Johannes C.C.M. in 't Veen ${ }^{5,6}$, Bruno H. Stricker ${ }^{2,7}$ and Guy G.O. Brusselle (1) ${ }^{1,2,6}$
}

${ }^{1}$ Dept of Respiratory Medicine, Ghent University Hospital, Ghent, Belgium. ${ }^{2}$ Dept of Epidemiology, Erasmus MC - University Medical Center Rotterdam, Rotterdam, The Netherlands. ${ }^{3}$ Dept of Bioanalysis, Ghent University, Ghent, Belgium. ${ }^{4}$ Dept of Medical Informatics, Erasmus MC - University Medical Center Rotterdam, Rotterdam, The Netherlands. ${ }^{5}$ Dept of Respiratory Medicine, Franciscus Gasthuis \& Vlietland, Rotterdam, The Netherlands. ${ }^{6}$ Dept of Respiratory Medicine, Erasmus MC - University Medical Center Rotterdam, Rotterdam, The Netherlands. ${ }^{7}$ Dept of Internal Medicine, Erasmus MC - University Medical Center Rotterdam, Rotterdam, The Netherlands.

Corresponding author: Guy G.O. Brusselle (guy.brusselle@ugent.be)

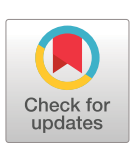

Copyright @The authors 2021

This version is distributed under the terms of the Creative Commons Attribution Non-Commercial Licence 4.0. For commercial reproduction rights and permissions contact permissions@ersnet.org

This article has supplementary material available from openres.ersjournals.com

Received: 22 Feb 2021 Accepted: 9 April 2021

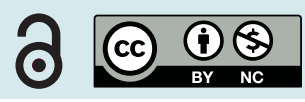

Shareable abstract (@ERSpublications)

Most middle-aged and older adults with asthma suffer from at least one severe exacerbation. Risk factors are previous exacerbations, use of SABA without concomitant controller medication, respiratory complaints, obesity, airway obstruction and depression. https://bit.ly/3gcTO6t

Cite this article as: de Roos EW, Lahousse L, Verhamme KMC, et al. Incidence and predictors of asthma exacerbations in middle-aged and older adults: the Rotterdam Study. ERJ Open Res 2021; 7: 00126-2021 [DOI: 10.1183/23120541.00126-2021].

\section{Abstract}

Aim The aim of this study was to investigate occurrence and determinants of asthma exacerbations in an ageing general population.

Methods Subjects aged 45 years or above with physician-diagnosed asthma in the Rotterdam Study, a population-based prospective cohort from January 1991 to May 2018, were assessed for asthma exacerbations. Exacerbations were defined as acute episodes of worsening asthma treated with oral corticosteroids. Cox proportional hazards analysis was used to investigate risk factors for a future exacerbation.

Results Out of 763 participants with asthma (mean age 61.3 years, 69.2\% female), 427 (56.0\%) experienced at least one exacerbation, in a mean follow-up time of 13.9 years. The mean annual exacerbation rate was 0.22. Most exacerbations occurred during winter months. Risk factors for exacerbations were a history of previous exacerbations (HR 4.25; 95\% CI 3.07-5.90, p<0.001)), respiratory complaints (HR 2.18; 95\% CI 1.48-3.21, p<0.001), airflow obstruction (HR 1.52; 95\% CI 1.07-2.15, $\mathrm{p}=0.019$ ), obesity (HR 1.38; 95\% CI 1.01-1.87, p=0.040) and depressive symptoms (HR 1.55; 95\% CI 1.05-2.29, $\mathrm{p}=0.027$ ). Compared to those not using respiratory medication, we observed higher hazard ratios for those on short-acting $\beta_{2}$-agonists (SABA, i.e. rescue medication) only (HR 3.08, 95\% CI 1.61-5.90, $\mathrm{p}=0.001$ ) than those on controller medication (HR 2.50, 95\% CI 1.59-3.92, $\mathrm{p}<0.001$ ).

Conclusion Many older adults with asthma suffer from at least one severe exacerbation. Previous exacerbations, use of SABA without concomitant controller medication, respiratory complaints, obesity, airway obstruction and depression are independent risk factors for exacerbations.

\section{Introduction}

Asthma is a common chronic respiratory disease, with a global prevalence in adults of 4.3\% [1]. Patients suffer from variable airflow obstruction resulting in respiratory symptoms, such as attacks of dyspnoea, limitation of activity [2] and impaired quality of life [3]. This burden of asthma is most substantial in those over 45 years of age (compared to the 18- to 45-year-olds), although this age group is comparatively understudied $[1,4]$. As there is no cure for asthma yet, treatment relies on achieving control of symptoms and the prevention of exacerbations [5].

A severe exacerbation is defined by the American Thoracic Society (ATS)/European Respiratory Society (ERS) as the need for systemic corticosteroids [6]. Exacerbations are responsible for a high human and 
financial burden (such as loss of working days and medical costs), and are the prelude to asthma-related emergency care, morbidity and even death [5, 7-10]. Fortunately, not all afflicted individuals with asthma suffer from exacerbations, which is more frequent in patients with severe asthma [9, 11-13]. Nevertheless, since patients with mild asthma are most prevalent in the general population, they still account for 30 to $40 \%$ of severe asthma exacerbations [14].

As most studies focus on specific patient cohorts, long-term follow-up data on asthma exacerbations in the general population are scarce, especially in the aforementioned groups of middle-aged and older subjects with mild-to-moderate asthma. Therefore, we studied the incidence of, and risk factors for, asthma exacerbations in middle-aged and older patients with asthma within a population-based sample with long-term follow-up.

Methods

Setting

All subjects participated in the Rotterdam Study, a prospective population-based cohort study that started in 1990 [15]. The objective of the Rotterdam Study is to investigate chronic diseases in the elderly. In 2000, the original cohort (RS-I; $n=7983$ ) was extended with a second cohort (RS-II; $n=3011$ ), and in 2006 with a third cohort (RS-III; $n=3932$ ). This resulted in a total study population of 14926 subjects (6103 men and 8823 women) aged 45 years and over (supplementary figure S1). An extensive set of examinations is performed every 3-5 years at the research facility, including biometrics, laboratory analyses, spirometry and cardiovascular investigations. Information on medication use is obtained from pharmacy-filled prescriptions from seven connected pharmacies in the region that serve $>95 \%$ of the study participants [15]. The Rotterdam Study was approved by the Medical Ethics Committee and by the Netherlands Ministry of Health [16]. All participants provided written informed consent to participate in the study.

\section{Asthma and follow-up}

Asthma cases were defined as participants with a physician’s diagnosis of asthma reported in their medical file. The accuracy of diagnosis has been validated, as described in detail elsewhere [17]. Subjects without informed consent to access their medical files or without any medication data were excluded. Follow-up time for prevalent asthma cases started at study entrance, whereas in subjects with incident asthma follow-up started at the date of asthma diagnosis. In both prevalent and incident cases of asthma, the follow-up time was calculated until the first exacerbation, death, lost to follow-up or end of the study period, whichever came first (flowchart provided in supplementary figure S2). Participants with COPD, defined as spirometry-confirmed airway obstruction in the absence of physician-diagnosed asthma, were excluded [18].

\section{Assessment of exacerbations}

Exacerbations were defined as a worsening of asthma symptoms that required treatment with systemic corticosteroids, conforming to the ATS/ERS statement on severe exacerbations [6]. Courses of corticosteroids separated by 1 week or more were regarded as separate exacerbations, in line with an earlier consensus statement [8]. In participants with both asthma and any use of respiratory medication, cases of oral use of prednisone, prednisolone or hydrocortisone prescriptions in a dose of 20-50 mg for a duration of 3 to 21 days were extracted from pharmacy records and marked as an asthma exacerbation. These criteria were based on the manual validation of a subset of $12 \%$ from all $(n=8581)$ oral, intramuscular and intravenous corticosteroid prescriptions from 1991 until 2016 in the medical files to determine the indication of the prescription. In this validation, $78.5 \%$ were prescribed for an asthma exacerbation, while most other indications were polymyalgia rheumatica, joint pain, skin disorders, gout and sinusitis. Further refinement entailed the selection of oral prednisone, prednisolone and hydrocortisone combined with dose and duration criteria. This raised the accuracy to $94 \%$ in our exacerbation validation set.

Exacerbation rate was calculated as the number of exacerbations/years of asthma follow-up time. Frequent exacerbators were defined as participants with an annual exacerbation rate of one or more.

Measurements and covariates

Spirometry (without reversibility testing) was performed by trained paramedical personnel according to the ATS/ERS guidelines [15]. Predicted values were determined according to the Global Lung Initiative (GLI) values 2012 [19]. Airway obstruction was defined as a forced expiratory volume in $1 \mathrm{~s}\left(\mathrm{FEV}_{1}\right) /$ forced vital capacity ratio $<0.7$.

COPD within the physician-diagnosed asthma cohort was defined as a COPD diagnosis next to an asthma diagnosis in the medical file. Smoking status was classified as never, former or current smoker. Blood 
eosinophil level was measured cross-sectionally and on indication by the general practitioner (GP). The value closest to the study baseline was used in the current study. Blood eosinophil measurements during concomitant oral steroid use were excluded. Hypertension was defined as a systolic blood pressure (SBP) $\geqslant 140 \mathrm{mmHg}$ or a diastolic blood pressure (DBP) $\geqslant 90 \mathrm{mmHg}$ or use of antihypertensive medication [20]. Type 2 diabetes mellitus was defined as a fasting blood glucose $\geqslant 7.0 \mathrm{mmol} \cdot \mathrm{L}^{-1}$, a nonfasting blood glucose $\geqslant 11.1 \mathrm{mmol} \cdot \mathrm{L}^{-1}$ or the use of blood glucose lowering medication [21]. Depressive symptoms were assessed with the validated Center for Epidemiologic Studies Depression Scale (CES-D), with scores ranging from 0 (no symptoms) to 60 (many symptoms); a cut-off score of 16 is commonly used and has acceptable screening accuracy for detecting major depression [22]. Osteoporosis was defined according to World Health Organisation (WHO) criteria as a T-score $\leqslant-2.5$.

Respiratory medication (as derived from pharmacy-filled prescriptions) was categorised based on the Anatomic Therapeutic Chemical classification (ATC) codes. The prescribed daily dose of each inhaled corticosteroid (ICS) was expressed in standardised defined daily doses according to the ATC/defined daily dose (DDD) system of the World Health Organisation (DDDs). ICS exposure was assessed based on pharmacy dispensing data on start of and during the follow-up period. Subjects were considered ICS users if their prescribed ICS covered $80 \%$ or more of their total follow-up time. For the calculation of the exacerbation hazards we classified the medication use of the past year (as predictor) into controller (ICS, long-acting $\beta 2$-agonists (LABA)+ICS, leukotriene receptor antagonist (LTRA), nedocromil and anti-allergics), rescue (short-acting muscarinic antagonists (SAMA), short-acting $\beta 2$-agonists (SABA)) or total medication (controller+rescue medication) [23]. Controller to total ratio was calculated as the total number of controller devices/the total number of respiratory medications.

\section{Statistical analysis}

This study is composed of two parts. In the first part, we calculated the incidence rate of asthma exacerbations and the exacerbation-free survival time during the entire follow-up within the Rotterdam Study. Descriptive characteristics were reported as mean (standard deviation) for continuous variables and as count (\%) for categorical variables. Survival curves for exacerbation-free time during follow-up were computed with the Survival package in R [24, 25].

In the second part, we studied risk factors for exacerbations in a sub-cohort, namely all participants with asthma in the fifth examination round and onwards (RS-I-5, RS-II-3 and RS-III-2, supplementary figure S1). This examination round offered more precise examinations including spirometry than the measurements taken at baseline from 1989 onwards and was therefore better suited to examine risk factors for future exacerbations. Baseline measures for these analyses were taken between 2009 and 2013, and follow-up was complete until June 2018.

The relationship between potential risk factors and the time to first exacerbation was analysed using a multi-variable Cox proportional hazard model. Variables of interest were derived from the literature [9, 11, 12]. Variables were tabulated as crude hazard ratios (HRs) and as age- and sex-adjusted HRs. Proportional hazards were inspected using plots and the proportional hazard assumption test from the Survival package in $\mathrm{R}[24,25]$.

Possible interaction with sex, based on earlier reports, was investigated and when reported as significant, followed by a stratified analysis [26]. Missing values were reported as such, without use of imputation techniques, as baseline missing data were not deemed missing at random. Statistical significance was set at 5\%. Data were analysed using SPSS Statistics (IBM, version 24.0) and R (The R Foundation for Statistical Computing, version 3.5.1).

Results

Incidence rate of severe asthma exacerbations

Patient characteristics of asthma subjects stratified by exacerbation status

Within the Rotterdam Study, there were 778 subjects with doctor-diagnosed asthma and full informed consent to extract information from their medical files. Of these, 763 had medication data available and were included in the analyses. Mean age of the population at start of follow-up was 61.3 years ( $\mathrm{SD}=8.3$; age range $=45.8$ to 91.2 years), with $69.2 \%$ women. The majority (i.e. 618 subjects; $81 \%$ ) had adult-onset asthma. The total asthma follow-up time was 9198.8 person years (mean duration of follow-up: 12.1 years, range 0.6 to 28.6 years). In that time period, 1832 exacerbations were recorded in 427 subjects (56.0\%). Baseline characteristics of study participants, stratified by exacerbation status, are shown in table 1 . 


\section{TABLE 1 Characteristics of study participants, stratified by exacerbation status}

\begin{tabular}{|c|c|c|}
\hline & $\begin{array}{c}\text { Asthma subjects without } \\
\text { exacerbations }\end{array}$ & $\begin{array}{l}\text { Asthma subjects with } \\
\text { exacerbations }\end{array}$ \\
\hline Subjects $\mathrm{n}$ & 336 & 427 \\
\hline Females n (\%) & $226(67.3)$ & $302(70.7)$ \\
\hline Age years & $60.85 \pm 8.43$ & $61.67 \pm 8.21$ \\
\hline BMI & $28.20 \pm 4.61$ & $28.25 \pm 4.79$ \\
\hline \multicolumn{3}{|l|}{ Smoking status $\mathrm{n}(\%)$} \\
\hline Never & $105(31.5)$ & $156(36.6)$ \\
\hline Former & $188(56.5)$ & $213(50.0)$ \\
\hline Current & $40(12.0)$ & $57(13.4)$ \\
\hline Total follow-up years & $11.98 \pm 6.08$ & $14.69 \pm 6.22$ \\
\hline Follow-up years with asthma & $10.74 \pm 5.59$ & $13.09 \pm 5.83$ \\
\hline $\begin{array}{l}\text { Asthma onset age years, median } \\
\text { (IQR) }\end{array}$ & $55.53(45.25,64.63)$ & $58.17(45.71,67.20)$ \\
\hline Asthma onset $>40$ years $n(\%)$ & $274(81.5)$ & 341 (79.9) \\
\hline Total number exacerbations & & $4.48 \pm 5.59$ \\
\hline Exacerbation rate & & $0.40 \pm 0.53$ \\
\hline \multicolumn{3}{|l|}{ GINA class n (\%) } \\
\hline 0 & $14(4.17)$ & $0.01(4)$ \\
\hline 1 & $61(18.2)$ & $2.81(12)$ \\
\hline 2 & $71(21.1)$ & $9.13(39)$ \\
\hline 3 & $120(35.7)$ & $30.0(128)$ \\
\hline $4+5$ & $70(20.8)$ & $57.1(244)$ \\
\hline Blood eosinophils $10^{3} \mu \mathrm{L}^{-1}$ & $0.25 \pm 0.13$ & $0.28 \pm 0.19$ \\
\hline Hypertension n (\%) & $142(53.2)$ & $171(49.4)$ \\
\hline Coronary heart disease $\mathrm{n}(\%)$ & $13(3.9)$ & $20(4.7)$ \\
\hline Diabetes mellitus n (\%) & $38(11.3)$ & $39(9.1)$ \\
\hline Depressive symptoms $\mathbf{n}(\%)$ & $37(15.5)$ & $54(20.9)$ \\
\hline Osteoporosis n (\%) & $9(3.3)$ & $18(5.2)$ \\
\hline \multicolumn{3}{|c|}{$\begin{array}{l}\text { Data are presented as mean } \pm \text { SD, unless otherwise stated. BMI: body mass index; GINA: Global Initiative for } \\
\text { Asthma. Baseline characteristics of study participants stratified by exacerbation status. Data were missing in: } \\
\text { BMI ( } n=61) \text {, smoking status ( } n=14) \text {, eosinophils }(n=285) \text {, hypertension }(n=12) \text {, depressive symptoms }(n=278) \text { and } \\
\text { osteoporosis }(n=151) \text {. }\end{array}$} \\
\hline
\end{tabular}

\section{Exacerbation rate}

The mean annual exacerbation rate was 0.22 (sD 0.42). The majority of asthma exacerbators ( $\mathrm{n}=300$ of 427) had more than one exacerbation, and 47 participants (6.2\%) had 10 or more exacerbations. Thirty-one subjects had a mean annual exacerbation rate above 1.0 (i.e. $4.1 \%$ out of total population and $7.3 \%$ out of those with any exacerbations). These frequent exacerbators were younger ( 57.4 years), were more often current smokers (36\%), had more often depressive symptoms (37\%) and developed asthma at younger age compared to those without exacerbations and those with infrequent exacerbations (supplementary table SI). The evaluation of all exacerbations by month depicted a seasonal variation, with the majority of the exacerbations occurring during winter months, with a trough in the summer (figure 1).

\section{Time to severe exacerbation}

The median exacerbation-free survival time within the total follow-up time was 9.76 years (95\% CI 8.45-11.34) (figure 2). Sex did not significantly alter the median exacerbation-free survival time. When stratified by smoking status, current smokers had the shortest time to an exacerbation, followed by never-smoking patients with asthma and former smokers (median time to exacerbation 7.62, 9.88 and 11.20 years, respectively).

A sub-analysis in those subjects who developed asthma during the Rotterdam Study follow-up (n=246) (i. $e$. incident asthma cases) depicted the same pattern as in the overall asthma cohort. Here the median severe exacerbation-free survival time was 8.67 years (95\% CI 6.75-11.34) (supplementary figure S3). In this group $43(17.5 \%)$ had a severe asthma exacerbation within the first year of diagnosis. 


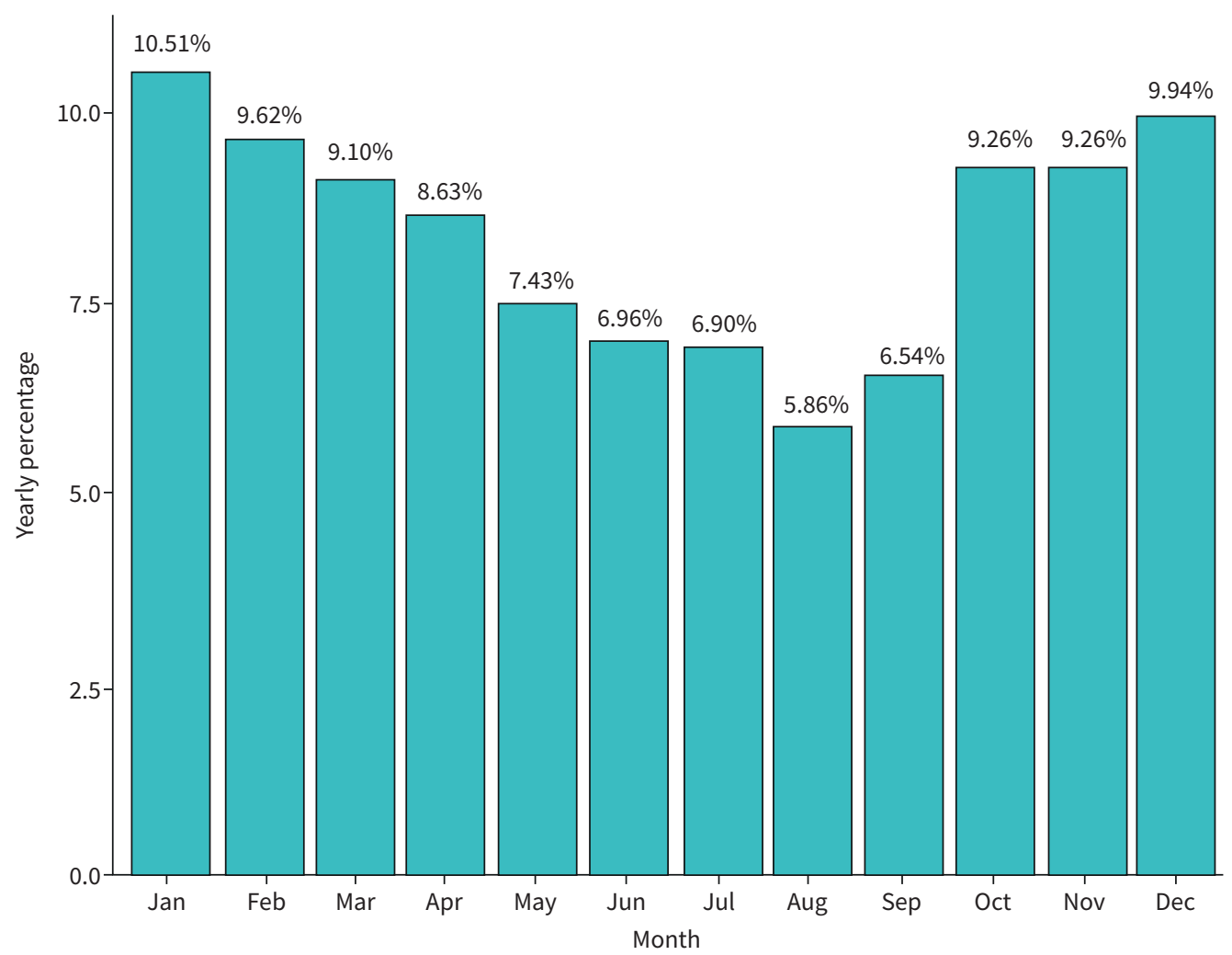

FIGURE 1 Monthly exacerbation percentage.

Risk factors for severe exacerbations

Patient characteristics at re-examination round

In the fifth examination round, 495 participants (8.1\% of total RS-5 $n=6110)$ with asthma were included in the analysis of risk factors associated with a severe asthma exacerbation. Mean age then was 69.3 years $(\mathrm{SD}=8.9$; age range $=51.8$ to 98.6 years), of whom $69.2 \%$ were women. Mean overall follow-up time was 4.2 years (range 0.5 to 9.1 years). During this follow-up, 177 participants had at least one severe exacerbation (baseline characteristics are provided in supplementary table SII).

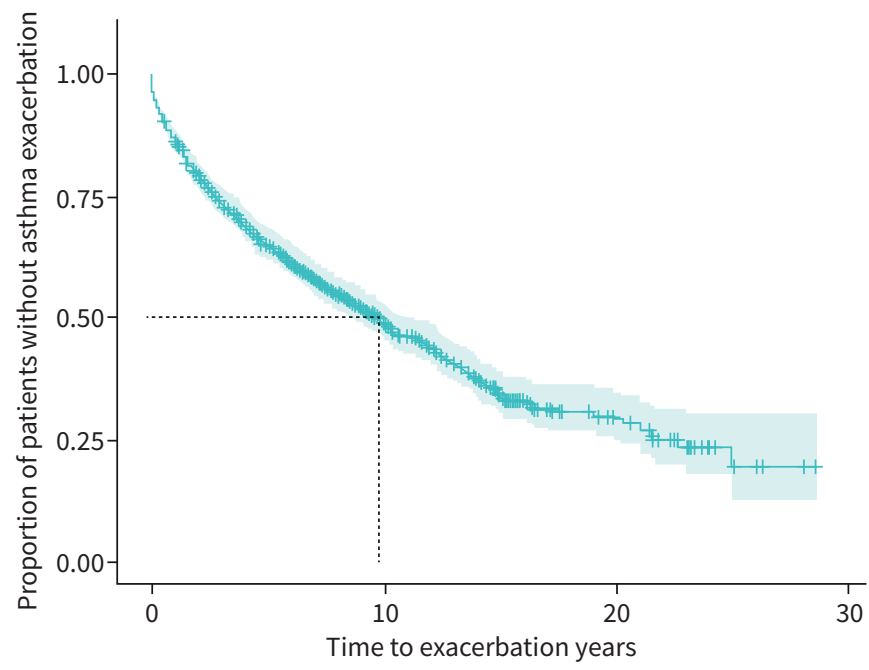

FIGURE 2 Time to severe exacerbation. Median survival time=9.8 years. 


\begin{tabular}{|c|c|c|c|}
\hline Determinant & Value & HR univariable ( $95 \% \mathrm{Cl}$, p-value) & HR model 1 (95\% Cl, p-value) \\
\hline Age years (mean $\pm s D)$ & $69.3 \pm 8.8$ & $0.98(0.96-1.00, p=0.048)$ & \\
\hline Female n (\%) & $345(69.7)$ & $0.97(0.70-1.33, p=0.842)$ & \\
\hline \multicolumn{4}{|l|}{ Smoking n (\%) } \\
\hline Never & $164(33.1)$ & Reference & Reference \\
\hline Former & $288(58.2)$ & $1.17(0.84-1.63, p=0.348)$ & $1.20(0.84-1.70, p=0.312)$ \\
\hline Current & $43(8.7)$ & $1.62(0.94-2.82, p=0.084)$ & $1.59(0.91-2.77, p=0.104)$ \\
\hline Smoking by sex interaction & & & $(p=0.0023)$ \\
\hline \multicolumn{4}{|l|}{ Obesity $^{\#} \mathrm{n}(\%)$} \\
\hline No & $319(64.4)$ & Reference & Reference \\
\hline Yes & $176(35.6)$ & $1.37(1.01-1.84, p=0.041)$ & $1.38(1.01-1.87, p=0.040)$ \\
\hline Previous exacerbation ${ }^{\circ} \mathrm{n}(\%)$ & $74(14.9)$ & $4.05(2.94-5.56, p<0.001)$ & $4.25(3.07-5.90, p<0.001)$ \\
\hline \multicolumn{4}{|l|}{ R03 medication ${ }^{+}$n (\%) } \\
\hline None & $165(33.3)$ & Reference & \\
\hline Rescue & $35(7.1)$ & $2.82(1.49-5.37, p=0.002)$ & $3.08(1.61-5.90, p=0.001)$ \\
\hline Controller & $167(33.7)$ & $2.45(1.57-3.82, p<0.001)$ & $2.50(1.59-3.92, p<0.001)$ \\
\hline Rescue+Controller & $128(25.9)$ & $4.50(2.90-7.00, p<0.001)$ & $4.42(2.82-6.94, p<0.001)$ \\
\hline R03 controller to total ratio & $0.8(0.3)$ & $0.79(0.50-1.26, p=0.324)$ & $0.77(0.48-1.23, p=0.276)$ \\
\hline \multicolumn{4}{|l|}{ Respiratory complaints ${ }^{\S} \mathrm{n}(\%)$} \\
\hline Wheezing & $323(65.8)$ & $2.08(1.45-2.96, p<0.001)$ & $2.07(1.44-2.97, p<0.001)$ \\
\hline Dyspnoea & $301(65.4)$ & $2.02(1.39-2.91, p<0.001)$ & $2.18(1.48-3.21, p<0.001)$ \\
\hline Chronic cough & $105(21.2)$ & $1.32(0.95-1.83, p=0.105)$ & $1.34(0.96-1.87, p=0.085)$ \\
\hline \multicolumn{4}{|l|}{ Lung function $\mathrm{n}(\%)$} \\
\hline Normal & $359(76.2)$ & Reference & Reference \\
\hline Restriction & $11(2.3)$ & $1.27(0.47-3.44, p=0.643)$ & $1.20(0.44-3.27, p=0.722)$ \\
\hline Obstruction & $101(21.4)$ & $1.57(1.11-2.20, p=0.010)$ & $1.52(1.07-2.15, p=0.019)$ \\
\hline $\mathrm{FEV}_{1} \%$ pred (mean $\left.\pm \mathrm{SD}\right)$ & $91.2 \pm 17.4$ & $0.98(0.97-0.99, p<0.001)$ & $0.97(0.97-0.99, p<0.001)$ \\
\hline COPD co-diagnosis n (\%) & $48(9.7)$ & $1.45(0.96-2.21, p=0.080)$ & $1.48(0.97-2.28, p=0.071)$ \\
\hline Depressive symptoms $^{f} \mathrm{n}(\%)$ & $72(14.7)$ & $1.50(1.02-2.19, p=0.038)$ & $1.55(1.05-2.29, p=0.027)$ \\
\hline Diabetes mellitus n (\%) & $44(9.0)$ & $1.18(0.70-1.97, p=0.530)$ & $1.12(0.66-1.91, p=0.678)$ \\
\hline Coronary heart disease $\mathrm{n}(\%)$ & $16(3.3)$ & $0.98(0.44-2.23, p=0.971)$ & $1.05(0.46-2.41, p=0.899)$ \\
\hline \multicolumn{4}{|c|}{$\begin{array}{l}\text { Univariate and model } 1 \text { are age and sex adjusted. } \mathrm{FEV}_{1} \text { : forced expiratory volume in } 1 \mathrm{~s} . \text { " : body mass index }>30 \text {; } \\
\text { ฯ: prior exacerbation in the last } 12 \text { months; }{ }^{+}: \text {any valid R03 prescription; }{ }^{\S}: \text { confirmative answer to "did you } \\
\text { suffer from wheezing/did you suffer from dyspnoea/did you cough daily for }>3 \text { months in the past } 2 \text { years?”; } \\
f: \text { Center for Epidemiologic Studies Depression scale }>16 \text {. Bold indicates statistical significance. }\end{array}$} \\
\hline
\end{tabular}

Determinants of severe exacerbations

Table 2 shows the HRs of a severe exacerbation per clinical determinant, adjusted for age and sex. A prior exacerbation in the past 12 months was associated with a significantly increased risk of subsequent exacerbations (HR 4.25, 95\% CI 3.07-5.90, p<0.001), as was airway obstruction (HR 1.52, 95\% CI 1.07-2.15, p=0.019), obesity (HR 1.38, 95\% CI 1.01-1.87, $\mathrm{p}=0.040$ ) and a high score of depressive symptoms (HR 1.55, 95\% CI 1.05-2.29, p=0.027). When participants reported complaints of either wheezing or dyspnoea at the study interview, this was also associated with a doubled risk (HR 2.07, 95\% CI 1.44-2.97, $\mathrm{p}<0.001$ and HR 2.18, 95\% CI 1.48-3.21, $\mathrm{p}<0.001$, respectively). A concomitant diagnosis of COPD next to asthma did not yield significant higher exacerbation rates.

Smoking behaviour as risk factor for exacerbation was different in males and females ( $\mathrm{p}$-value for the age-adjusted interaction=0.002). Therefore, we performed a sex-stratified analysis corrected for age and $\mathrm{FEV}_{1}$. Woman with asthma and persistent smoking had higher HRs (HR 2.34, 95\% CI 1.26-4.33 p=0.007) compared to women who had stopped smoking (HR 2.06, 95\% CI 1.33-3.18, p=0.001) or never smoked (reference), whereas in males this direction was not observed (supplementary figure S4).

\section{Asthma medication and exacerbations}

With regard to respiratory medication, with "no use" as reference category, we observed higher HRs for those on short-acting bronchodilator (i.e. SABA rescue) medication only than those on controller medication only (HR 3.08, 95\% CI 1.61-5.90, p=0.001 and HR 2.50, 95\% CI 1.59-3.92, $\mathrm{p}<0.001$, respectively). Subjects using both control and rescue medications had the highest risk. 


\begin{tabular}{|c|c|c|c|c|}
\hline & \multirow[b]{2}{*}{ n (\%) } & \multirow{2}{*}{$\begin{array}{c}\text { Severe exacerbation last year } \\
\text { HR age }+ \text { sex adjusted } \\
(95 \% \mathrm{Cl}, \mathrm{p} \text {-value })\end{array}$} & \multicolumn{2}{|c|}{ No severe exacerbation last year" } \\
\hline & & & n (\%) & $\begin{array}{l}\text { HR age }+ \text { sex adjusted } \\
\quad(95 \% \mathrm{Cl}, \mathrm{p} \text {-value })\end{array}$ \\
\hline \multicolumn{5}{|l|}{ R03 medication } \\
\hline None & $5(6.8)$ & \multicolumn{3}{|c|}{$160(38.0)$} \\
\hline Rescue & $5(6.8)$ & $4.78(1.10-20.82, p=0.037)$ & $30(7.1)$ & $2.06(0.96-4.44, p=0.065)$ \\
\hline Controller & $20(27.0)$ & $1.04(0.30-3.65, p=0.953)$ & $147(34.9)$ & $2.27(1.41-3.67, p=0.001)$ \\
\hline Controller and rescue & $44(59.5)$ & $1.09(0.33-3.56, p=0.889)$ & $84(20.0)$ & $3.64(2.16-6.16, p<0.001)$ \\
\hline \multicolumn{5}{|l|}{ Controller to total ratio } \\
\hline Controller/controller+rescue & $74(100)$ & $0.26(0.09-0.76, p=0.014)$ & $421(100)$ & $1.13(0.61-2.10, p=0.691)$ \\
\hline
\end{tabular}

When data were stratified according to the exacerbation status in the previous year, we observed, especially in asthma subjects with a previous exacerbation, a significant inverse relationship between the controller to total (controller+reliever) asthma medication ratio and the hazard of a subsequent exacerbation (HR age and sex adjusted: 0.26, 95\% CI 0.09-0.76, p=0.014) (table 3).

\section{Discussion}

Asthma is a heterogeneous disease as is reflected in the different levels of symptom control and the occurrence of exacerbations. In this study, encompassing a population-based asthma cohort of middle-aged and older patients, we demonstrated that although the majority of older adults with asthma have infrequent exacerbations, $17.5 \%$ of subjects with incident (thus late-onset) asthma suffer from at least one severe exacerbation within the first year of diagnosis. Moreover, we showed that treatment of middle-aged and older asthmatic patients with short-acting bronchodilators alone (i.e. SABA use without concomitant ICS) is associated with a significantly increased risk of severe exacerbations, whereas a higher ratio of controller to total asthma medications is protective.

Our results show that $56 \%$ of asthma patients suffer at least one exacerbation during follow-up even though most subjects are regarded as having mild asthma. The annual rate of moderate to severe exacerbations in our study is 0.22. In comparison, in the SYGMA trials, enrolling adolescent and adult patients with mild asthma, an annual exacerbation rate of 0.20 was reported in the as-needed SABA (terbutaline) group versus 0.07 in the as-needed budesonide-formoterol fixed dose combination (FDC) group [27]. Mean age in the SYGMA trial, however, was 41.0 years, which is significantly younger than in the Rotterdam Study (mean age at enrolment 61.7 years). Similarly, a retrospective study including $>400000$ subjects from the UK and USA by SuRUKi et al. [9], found a mean annual rate of exacerbations of 0.16 in the USA cohort (mean age 38 years) and 0.11 in the UK set (mean age 45 years). In contrast with these findings is the high rate of 0.94 (9.4 per 10 person-years in the original paper) that Bцоом et al. [11] report in their population of 55 years and older, stressing the fact that comparable data with our middle-aged and older cohort is scarce.

The seasonal distribution of asthma exacerbations in our data showed a peak in winter and a nadir from July to September. This resembles more the pattern that is seen in COPD than in children and young adults with asthma in whom often a peak is reported in February and September [28-30]. A recent study by SATIA et al. [31] documents the respiratory viral pattern throughout the year and demonstrates a biphasic pattern for asthma exacerbations mainly discernible in children, while this pattern becomes less pronounced in those above 70 years of age. COPD has a nadir in August and has a fairly comparable pattern with the overall respiratory tract infections graph. This may indicate that exacerbation risk factors attributed to COPD (such as viral respiratory tract infections and moist weather) are also important in older adult asthma, whereas aeroallergen-exposure-induced exacerbations become less important. Comparing the seasonal pattern of asthma exacerbations in the middle-aged and older asthma patients in the Rotterdam Study with publicly available graphs on Dutch pollen counts [32], our data most closely resemble the data on viral respiratory tract infections and lack a clear representation of aeroallergen-induced exacerbations. This observation is in line with increased proportions of nonallergic mechanisms and neutrophilic and noneosinophilic disease in late-onset asthma [33]. 


\section{Risk factors for exacerbation}

Current asthma care would immensely benefit from a reliable prediction model for asthma exacerbations. Various attempts have been made, but so far previous healthcare use or a previous exacerbation remains the strongest predictor, and our data are no exception. A hurdle in the formation of a prediction model is often the lack of cross-validation or discrepancy between the outcome measures. In a recent external validation of multiple prediction models, lung function, complaints and previous healthcare use were common themes [12], and this is in line with our results. However the prediction of exacerbations is also complicated by the high variation between the exacerbation intervals as reported by BLoom et al. [13]. By using a Cox proportional hazard model instead of a logistic regression with Poisson modelling, we aimed to account for this variability.

Others have reported female sex as an independent risk factor for exacerbations, and this effect is reported to be partially mediated by obesity [34, 35]. In our data this effect is driven by the interaction between smoking and sex, independent of obesity, where female current smokers are at the highest risk [11, 36, 37]. As the number of male current smokers is small, we put forward the hypothesis that men who smoke, more often than women who smoke, unjustly, might get a GP diagnosis of COPD.

The use of medication is of course an indicator of disease severity and should therefore be cautiously interpreted. Several randomised controlled clinical trials have demonstrated the effectiveness of ICS/ formoterol as-needed therapy in mild asthma, preventing exacerbations as compared with SABA use only (e.g. novel START study and SYGMA trials [27, 38, 39]). Today, the Global Initiative for Asthma (GINA) no longer recommends the use of short-acting bronchodilators alone in adolescents and adults with asthma [40]. In contrast, GINA now recommends ICS-containing treatments in mild asthma, either as daily controller treatment or as anti-inflammatory reliever. Our real-world observations in a population-based sample of older patients with (mainly mild) asthma support this treatment strategy.

Finally, we replicated the finding that depressive symptoms in asthma subjects are independently predictive of future exacerbations. This finding is intriguing as it can be regarded as a treatable trait, apart from the fact that the causal direction is not certain [37, 41-44].

\section{Strengths and limitations}

The strength of the Rotterdam Study is its prospective, population-based design with long follow-up time that enabled us to study both those with prevalent asthma, as well as new incident asthma cases during follow-up. In a real-life setting our data provide insight into the proportion of older adults suffering an exacerbation within a year after diagnosis.

Another strength is the assessment and validation of exacerbations to minimise misclassification. Several methods to assess oral corticosteroid (OCS) use as proxy for an exacerbation are reported in the literature, e.g. all OCS within 2 weeks of an asthma disease code in the medical file [9], all prescriptions $\leqslant 300 \mathrm{mg}$ [11], any OCS equivalent to $20 \mathrm{mg}$ prednisone for 3-28 days [9] or OCS courses during at least 3 days [45]. Most studies include (young) adults up to age of 50 years, while in an ageing population, other reasons for corticosteroid prescriptions also become more eminent, e.g. polymyalgia rheumatica or gout.

This study has limitations. Our data set lacks information on hospitalisations, and this could have led to an underestimation of the true occurrence of exacerbations. However, in the Netherlands the mean duration of asthma-related hospital stay was 4.9 days in 2011, whereas the guidelines for the duration of OCS exceeded 7 days [46]. Therefore, subjects that had to continue OCS after discharge will be included in our data. Also, as stated before, inferences based on medication use can lead to confounding by disease severity. As our real-life observations are in line with previous large trials, we consider these data of relevance to this study. We acknowledge that the lack of specialist clinical parameters (FeNO, bronchial hyperresponsiveness) and patient-reported outcomes (e.g. Asthma Control Questionnaire (ACQ), Asthma Control Test (ACT) or Asthma Quality of Life Questionnaire (AQLQ)) due to the population-based setting limits the current study, mainly with regard to the inference of exacerbation-associated risk factors. Finally, one can dispute our inclusion of participants with the combination of a valid asthma diagnosis with concomitant airway obstruction and a history of smoking. As this reflects the real-life population, we deemed this decision reasonable, as we provided insight into spirometry values as well as correcting for smoking and airway obstruction in our inferences. 
Striving for good asthma control is warranted, especially in patients with late-onset asthma, as $17.5 \%$ of these patients experience at least one severe exacerbation within the first year after diagnosis. Risk factors for exacerbations in this cohort of middle-aged and elderly patients are a history of previous exacerbations, lack of controller medication use, subjective respiratory complaints, obesity, airway obstruction and depression.

Conflict of interest: E.W. de Roos has nothing to disclose. L. Lahousse has nothing to disclose. K.M.C. Verhamme works for a research group that received/receives unconditional research grants from Yamanouchi, Pfizer-Boehringer Ingelheim, Novartis, GSK, Chiesi and Amgen. G-J. Braunstahl reports grants from GSK, AstraZeneca, Chiesi and Teva, personal fees from Novartis and Sanofi, and grants and personal fees from ALK Abello, outside the submitted work. J.C.C.M. in 't Veen reports faculty grants from Teva, Chiesi, Boehringer Ingelheim and AstraZeneca, and speaker bureau and advisory board fees from Sanofi, outside the submitted work. B.H. Stricker has nothing to disclose. G.G.O. Brusselle reports advisory board fees from Amgen, Boehringer Ingelheim, Novartis, Sanofi and Teva, and advisory board and lecture fees from AstraZeneca, Chiesi and GlaxoSmithKline, outside the submitted work.

\section{References}

1 The Global Asthma Report 2014. The Global Asthma Report 2014 - Global Burden of Disease due to Asthma, Auckland. New Zealand, Global Asthma Network, 2014.

2 Vermeulen F, Garcia G, Ninane V, et al. Activity limitation and exertional dyspnea in adult asthmatic patients: what do we know? Respir Med 2016; 117: 122-130.

3 Papi A, Brightling C, Pedersen SE, et al. Asthma. Lancet 2018; 391: 783-800.

4 Braman SS. The global burden of asthma. Chest 2006; 130: 4S-12S.

5 Global Initiative for Asthma. Global Strategy for Asthma Management and Prevention, 2019. Available from: http://www.ginasthma.org Date last accessed: 2 February 2021.

6 Reddel HK, Taylor DR, Bateman ED, et al. American Thoracic Society/European Respiratory Society Task Force on Asthma Control and Exacerbations. An Official American Thoracic Society/European Respiratory Society statement: asthma control and exacerbations: standardising endpoints for clinical asthma trials and clinical practice. Am J Respir Crit Care Med 2009; 180: 59-99.

7 Sullivan PW, Ghushchyan VH, Campbell JD, et al. Measuring the cost of poor asthma control and exacerbations. J Asthma 2017; 54: 24-31.

8 Fuhlbrigge A, Peden D, Apter AJ, et al. Asthma outcomes: exacerbations. J Allergy Clin Immunol 2012; 129: S34-S48.

9 Suruki RY, Daugherty JB, Boudiaf N, et al. The frequency of asthma exacerbations and healthcare utilisation in patients with asthma from the UK and USA. BMC Pulm Med 2017; 17: 74.

10 Mukherjee M, Stoddart A, Gupta RP, et al. The epidemiology, healthcare and societal burden and costs of asthma in the UK and its member nations: analyses of standalone and linked national databases. BMC Med 2016; 14: 113.

11 Bloom Cl, Nissen F, Douglas IJ, et al. Exacerbation risk and characterisation of the UK's asthma population from infants to old age. Thorax 2018; 73: 313-320.

12 Loymans RJB, Debray TPA, Honkoop PJ, et al. Exacerbations in adults with asthma: a systematic review and external validation of prediction models. J Allergy Clin Immunol Pract 2018; 6: 1942-1952.

13 Bloom Cl, Palmer T, Feary J, et al. Exacerbation patterns in adults with asthma in England. A population-based study. Am J Respir Crit Care Med 2019; 199: 446-453.

14 Dusser D, Montani D, Chanez P, et al. Mild asthma: an expert review on epidemiology, clinical characteristics and treatment recommendations. Allergy 2007; 62: 591-604.

15 Ikram MA, Brusselle GGO, Murad SD, et al. The Rotterdam study: 2018 update on objectives, design and main results. Eur J Epidemiol 2017; 32: 807-850.

16 Hofman A, Brusselle GG, Darwish Murad S, et al. The Rotterdam study: 2016 objectives and design update. Eur J Epidemiol 2015; 30: 661-708.

17 de Roos EW, Lahousse L, Verhamme KMC, et al. Asthma and its comorbidities in middle-aged and older adults: the Rotterdam study. Respir Med 2018; 139: 6-12.

18 Terzikhan N, Verhamme KM, Hofman A, et al. Prevalence and incidence of COPD in smokers and non-smokers: the Rotterdam study. Eur J Epidemiol 2016; 31: 785-792.

19 Global Lung Function Initiative. European Respiratory Society, 2018. www.erseducation.org/guidelines/globallung-function-initiative Date last accessed: 9 June 2021.

20 Mancia G, Fagard R, Narkiewicz K, et al. 2013 ESH/ESC guidelines for the management of arterial hypertension: the Task Force for the Management of Arterial Hypertension of the European Society of Hypertension (ESH) and of the European Society of Cardiology (ESC). Eur Heart J 2013; 34: 2159-2219. 
21 Ligthart S, van Herpt TT, Leening MJ, et al. Lifetime risk of developing impaired glucose metabolism and eventual progression from prediabetes to type 2 diabetes: a prospective cohort study. Lancet Diabetes Endocrinol 2016; 4: 44-51.

22 Vilagut G, Forero CG, Barbaglia G, et al. Screening for depression in the general population with the Center for Epidemiologic Studies Depression (CES-D): a systematic review with meta-analysis. PLoS One 2016; 11: e0155431.

23 WHO Collaborating Centre for Drug Statistics Methodology. NIPH (fhi.no). Published 7 June 2014. www.fhi.no/ en/hn/drug/who-collaborating-centre-for-drug-statistics-methodology/ Date last accessed: 9 February 2021; date last updated: 14 August 2018.

24 Therneau TM, Grambsch PM. Modeling Survival Data: Extending the Cox Model. New York, Springer Science \& Business Media, 2013.

25 Therneau TM. A package for survival analysis in s. 2015, version 2.38. https://CRAN.R-project.org/ package=survival Date last accessed: 9 June 2021; date last updated: 26 April 2021.

26 Sood A, Qualls C, Schuyler M, et al. Adult-onset asthma becomes the dominant phenotype among women by age 40 years. The Longitudinal Cardia Study. Ann Am Thorac Soc 2013; 10: 188-197.

27 O'Byrne PM, FitzGerald JM, Bateman ED, et al. Inhaled combined budesonide-formoterol as needed in mild asthma. N Engl J Med 2018; 378: 1865-1876.

28 Rabe KF, Fabbri LM, Vogelmeier C, et al. Seasonal distribution of COPD exacerbations in the prevention of exacerbations with tiotropium in COPD trial. Chest 2013; 143: 711-719.

29 Staton TL, Arron JR, Olsson J, et al. Seasonal variability of severe asthma exacerbations and clinical benefit from lebrikizumab. J Allergy Clin Immunol 2017; 139: 1682-1684.

30 Johnston NW, Sears MR. Asthma exacerbations. 1: epidemiology. Thorax 2006; 61: 722-728.

31 Satia I, Adatia A, Yaqoob S, et al. Emergency department visits and hospitalisations for asthma, COPD and respiratory tract infections: what is the role of respiratory viruses, and return to school in September, January and March? ERJ Open Res 2020; 6: 00593-02020.

32 Pollennieuws. https://pollennieuws.nl/ Date last updated: 2 April 2021; date last accessed: 4 February 2021.

33 Hirano T, Matsunaga K. Late-onset asthma: current perspectives. J Asthma Allergy 2018; 11: 19-27.

34 Haldar P, Pavord ID, Shaw DE, et al. Cluster analysis and clinical asthma phenotypes. Am J Respir Crit Care Med 2008; 178: 218-224.

35 Han YY, Forno E, Celedón JC. Sex steroid hormones and asthma in a nationwide study of U.S. adults. Am J Respir Crit Care Med 2020; 201: 158-166.

36 Kang HR, Song HJ, Nam JH, et al. Risk factors of asthma exacerbation based on asthma severity: a nationwide population-based observational study in South Korea. BMJ Open 2018; 8: e020825.

37 Price D, Wilson AM, Chisholm A, et al. Predicting frequent asthma exacerbations using blood eosinophil count and other patient data routinely available in clinical practice. J Asthma Allergy 2016; 9: 1-12.

38 Reddel HK, Busse WW, Pedersen S, et al. Should recommendations about starting inhaled corticosteroid treatment for mild asthma be based on symptom frequency: a post-hoc efficacy analysis of the start study. Lancet 2017; 389: 157-166.

39 Bateman ED, Reddel HK, O'Byrne PM, et al. As-needed budesonide-formoterol versus maintenance budesonide in mild asthma. N Engl J Med 2018; 378: 1877-1887.

40 Reddel HK, FitzGerald JM, Bateman ED, et al. Gina 2019: a fundamental change in asthma management: treatment of asthma with short-acting bronchodilators alone is no longer recommended for adults and adolescents. Eur Respir J 2019; 53: 1901046

41 Yohannes Abebaw M, Newman Mary, Kunik Mark E. Psychiatric collaborative care for patients with respiratory disease. Chest 2019; 155: 1288-1295.

42 McDonald VM, Hiles SA, Godbout K, et al. Treatable traits can be identified in a severe asthma registry and predict future exacerbations. Respirology 2019; 24: 37-47.

43 Gao YH, Zhao HS, Zhang FR, et al. The relationship between depression and asthma: a meta-analysis of prospective studies. PLoS One 2015; 10: e0132424.

44 Zhang L, Zhang X, Zheng J, et al. Co-morbid psychological dysfunction is associated with a higher risk of asthma exacerbations: a systematic review and meta-analysis. $J$ Thorac Dis 2016; 8: 1257-1268.

45 Chanoine S, Pin I, Sanchez M, et al. Asthma medication ratio phenotypes in elderly women. J Allergy Clin Immunol Pract 2018; 6: 897-906.e895.

46 Public Health Care.info. Asthma. www.volksgezondheidenzorg.info/onderwerp/astma Date last accessed: 10 October 2020. 